\title{
Teoría de la educación
}

Education theory / Teoria da educação

El declive de la educación de la voluntad: problemática y tendencias educativas

The Decline of Education of the Will: Educational Issues and Trends

O declínio da educação da vontade: problemáticas e tendências educativas

Alejandra Peñacoba-Arribas, Universidad Católica de Colombia, Colombia Dalia Santa Cruz-Vera, Universidad Católica de Colombia, Colombia 



\section{El declive de la educación de la voluntad: problemática y tendencias educativas}

\section{Alejandra Peñacoba-Arribas}

Universidad Católica de Colombia, Colombia

apenacoba@ucatolica.edu.co

\section{Dalia Santa Cruz-Vera}

Universidad Católica de Colombia, Colombia

djsantacruz@ucatolica.edu.co

\section{Resumen}

Este artículo es resultado de una investigación documental sobre la educación de la voluntad. En él se abordan las problemáticas asociadas a la formación de la voluntad en la sociedad actual, su fundamentación teórica y las tendencias educativas en este ámbito. Entre los factores que parecen incidir en el declive de la educación de la voluntad se encuentran un extendido permisivismo educativo; el fomento de una cultura emotiva, que privilegia el sentimiento por encima de la razón y de la voluntad, así como el facilismo y la mediocridad frutos de una "voluntad débil". Para la educación de la voluntad, debe tenerse en cuenta su doble dimensión cognitiva y motivacional. Lo valioso motiva el obrar humano. Los educadores deben tenerlo en cuenta al diseñar estrategias pedagógicas con ese fin. A grandes ideales corresponden grandes respuestas. Finalmente, se presentan tres tendencias educativas actuales que aúnan diversas iniciativas en orden a la formación de la voluntad: educación de la voluntad, educación del carácter y educación moral.

\section{Palabras clave}

Educación del carácter; educación moral; educación de la voluntad; educación en los valores; teoría de la educación (Fuente: Tesauro de la Unesco).

\footnotetext{
Recepción: 2015-10-13 | Envío a pares: 2016-07-26 | Aceptación por pares: 2016-08-23 | Aprobación: 2016-08-30 DOI: 10.5294/edu.2016.19.3.7

Para citar este artículo / To reference this article / Para citar este artigo
}

Peñacoba-Arribas, A. y Santa Cruz-Vera, D. (2016). El declive de la educación de la voluntad: problemática y tendencias educativas. Educ. Educ., 19(3), 439-457. DOI: 10.5294/edu.2016.19.3.7 


\title{
The Decline of Education of the Will: Educational Issues and Trends
}

\begin{abstract}
This article is the result of a documentary research about the education of the will. The paper addresses issues related to the shaping of the will in current society, its theoretical background and the educational trends in this field. Among the factors that seem to affect the decline of the education of the will are: a widespread educational permissiveness; the enhancement of an emotional culture, which privileges feelings over reason and will, as well as a tendency to take the easy way out and the mediocrity resulting from a "weak will". Regarding education of the will, it is important to consider its double cognitive and motivational dimension. Valuable things motivate human behavior. Educators must consider this when designing pedagogical strategies with that purpose. Great ideals call for great answers. Finally, the paper presents three current educational trends that combine several initiatives regarding the shaping of will: education of the will, character education and moral education.
\end{abstract}

\section{Keywords}

Character Education; Moral Education; Education of the Will; Values Education; Education Theory (Source: Unesco Thesaurus). 


\section{O declínio da educação da vontade: problemáticas e tendências educativas}

Resumo

Este artigo é o resultado de uma pesquisa documental sobre a educação da vontade. Aqui, se abordam as problemáticas relacionadas à formação da vontade na sociedade atual, sua fundamentação teórica e as tendências educativas neste âmbito. Entre os fatores que parecem incidir no declínio da educação da vontade encontra-se uma extensa permissividade educativa; o fomento de uma cultura emotiva, que privilegia o sentimento acima da razão e da vontade, e a tendência a buscar a opção mais fácil e a mediocridade como resultado de uma "vontade fraca". Para a educação de vontade, deve-se ter em conta sua dupla dimensão cognitiva e motivacional. Aquilo que é valioso motiva o fazer humano. Os educadores devem considerá-lo quando desenham estratégias pedagógicas para este fim. Grandes ideais conduzem a grandes respostas. Finalmente, apresentam-se três tendências educativas atuais, que reúnem diferentes iniciativas, de conformidade com a formação da vontade: educação da vontade, educação do caráter e educação moral.

\section{Palavras-chave}

Educação do caráter; educação moral; educação da vontade: educação nos valores; teoria da educação (Fonte: Tesauro da Unesco). 
La juventud actual muere por comprender demasiado y querer poco.

Tomás Morales

\section{Introducción}

La educación tiene como finalidad contribuir al perfeccionamiento de la persona en todas sus dimensiones. Este crecimiento personal no estriba en el "tener más" sino en el "ser más", es decir en el desarrollo de los hábitos de la inteligencia y de la voluntad (Barrio, 2007) que lo capacitan para gestionar adecuadamente su libertad.

Se pueden considerar como pilares de una formación integral, la atención a las capacidades fundamentales de la persona: inteligencia, voluntad y afectividad. Lo que sintéticamente puede presentarse, siguiendo a Morales (2008), como: enseñar a pensar, a querer y a amar.

La educación de la voluntad consistiría, entonces, en este enseñar a querer el bien, es decir, en ayudar al educando a "ordenar los propios impulsos para ser capaz de tomar decisiones mediante el correcto uso de la propia libertad y llevarlas a término con responsabilidad", a saber "hacer frente a las dificultades y frustraciones aprendiendo a asumirlas como parte del proceso de desarrollo personal" (Risco, Sánchez y Urchaga, 2010, p. 42).

Si bien el proceso educativo debe atender a la persona en su totalidad, en este artículo prestaremos especial atención a la formación de la voluntad, a la forja del carácter del joven. Esto por la importancia que reviste el tema en orden a la perfección personal del educando y que viene siendo marginado en la pedagogía actual, especialmente en la educación superior (Marina, 1997). Y es que, dentro de una sociedad llamada "del conocimiento", con frecuencia se valora más el desarrollo intelectual que la vida virtuosa o esforzada, los logros académicos que la firmeza de carácter para elegir el bien y llevar adelante el propio proyecto de vida.
De ahí que el artículo aborde, en primer lugar, la importancia y urgencia de la educación de la voluntad en los jóvenes, señalando aquellos factores que inciden en su perfeccionamiento. En un segundo momento, se afronta su fundamentación teórica desde una visión personalista de la educación. Finalmente, se presentan algunas tendencias educativas actuales que subrayan la importancia del cultivo de la virtud para la educación de la voluntad y la plenitud personal.

\section{Importancia y urgencia de la educación de la voluntad}

Numerosos autores afirman que la educación de la voluntad es un elemento primordial e irrenunciable en la educación de la juventud, y se considera de vital importancia en la estructuración de la personalidad (García Hoz, 1987, 1988; Polaino-Lorente, 1988; Sacristán 1988; García Ramos, 1991; Jiménez, 1994; Jiménez y Sánz 2015; Quintana Cabanas, 1995; Otero, 1998; Barrio, 2007; Morales, 2008; Osorio, 2010; Rojas, 2010; Peñacoba, 2014).

Es esclarecedor el análisis que hace Abilio de Gregorio al respecto:

...una vida creadora demanda una personalidad en la que el sujeto se siente y es dueño de su voluntad. Puede la inteligencia indicar con absoluto claridad cuáles son los caminos que se han de seguir, pero si no se dispone de una instancia que señorea por encima de cualquier otra instancia a la hora de la decisión, se termina arrastrados por el convoy de los acontecimientos, de la opinión pública, de las mayorías, de las modas, etc. Sin una sólida voluntad no hay capacidad alguna de creación. La crisis de sentido a la que asistimos no es solamente consecuencia del eclipse de la verdad y de la obturación del entendimiento. Se manifiesta también en un llamativo apocamiento de la voluntad (2008, pp.304-305). 
Entre los factores influyentes en el declive que ha tenido en las últimas décadas la educación de la voluntad se puede señalar, en primer lugar, la instalación de un cierto permisivismo pedagógico dentro del ámbito educativo (Polaino-Lorente, 1988; Rojas, 2010). Para Polaino Lorente, por ejemplo, esto se debería en parte a una comprensión inadecuada de la voluntad en cuanto facultad humana espiritual. De ahí se desprende que las propuestas pedagógicas actuales, muchas veces, pongan un mayor énfasis en la "motivación" externa del estudiante por parte del educador y se desprecie o arrincone el necesario -e imprescindible- esfuerzo que debe realizar el educando dentro del proceso de aprendizaje.

Para autores como Vargas y González-Torres (2009), en la educación actual se conjuga el miedo a exigir con el fomento de una malentendida autoestima, conduciendo, muchas veces, a prácticas pedagógicas desenfocadas. Al respecto escriben:

El resultado de esto es un infundado temor a dañar el "self" del niño, lo cual se ha traducido en unas prácticas educativas y en una cultura con miedo a educar, a poner límites, a ejercer autoridad y en general a exigir algo que contradiga el ambivalente deseo del niño y del adolescente. Esto ha favorecido en los jóvenes el egocentrismo y el narcisismo, y paradójicamente la formación de una autoestima débil, inflada y frágil (p. 1383).

Es llamativo al respecto, por ejemplo, el título del libro de Aldo Naouri (2005) Padres permisivos, hijos tiranos, donde el autor expone la "frustración" precoz de los hijos como camino de educación.

En esta misma línea, Morales señala una cierta "atonía de la voluntad" presente en los jóvenes de hoy. Esta se configura como "una enfermedad de la voluntad que procede del miedo a comprometerse, del horror ante el esfuerzo". Al parecer, "nos cansamos de todo lo que emprendemos. Nos cuesta decidirnos a hacer algo" (2008, p. 437).
Frente a ese "permisivismo" presente en la sociedad, las instituciones educativas se presentan como aquellas que tienen la obligación de ayudar al educando a decidirse por el bien y la verdad, aunque suponga esfuerzo. Por el contrario, si todo se le permite al educando será porque no existen valores que proponer, o que nada se considera como bueno o como malo sino indiferente; ahora bien, como escribe Polaino-Lorente (1988), lo indiferente difícilmente podrá motivar la voluntad. En este punto, podemos constatar una estrecha relación entre permisivismo educativo y relativismo ético (Rojas, 2010).

En una reciente investigación se concluye que la ideología del permisivismo debe entenderse, más que como tolerancia, como indiferencia.

Esta actitud de indiferencia que se ha denominado como la ética de la neutralidad, mantiene que uno es más libre cuanto más alejado está de convicciones, de afecciones que le comprometan; esta neutralidad, cargada de indiferencia, aboca a una voluntad liberada de cualquier atadura y convertida en puro deseo (inclinación) (Gordillo, 2105, p. 62).

Las repercusiones pedagógicas pueden observarse en la tendencia a confundir el "querer" de la voluntad, con el "deseo" de la sensibilidad. De ahí que con facilidad se califique negativamente de "represión" el inhibir ciertas acciones demasiado espontáneas del educando, en orden a ayudarle a orientar su conducta a través de la reflexión personal (Polaino-Lorente, 1988). "Si la voluntad es entendida como deseo no tiene otra función que la toma de decisiones, la voluntad se convierte en algo vacío, el querer es un impulso ciego que se mueve por la inclinación a lo más placentero" escribirá Gordillo (2015, p. 62).

En la sociedad actual podemos observar un creciente instalarse de lo que se ha venido llamando "cultura del emotivismo y de la inmediatez", un cierto vivir para el placer del instante. Este emotivismo instalado en la sociedad actual, con su consecuente 
"vivir para el instante", es otro de los factores que parecen estar asociados al declive de formación de la voluntad de los jóvenes. Este emotivismo consiste en una valoración hipertrofiada del sentimiento, en detrimento muchas veces de la formación de la inteligencia y de la voluntad (Sellés, 2009).

El sentimental se ciñe de ordinario a las emociones sensibles, es decir, a las más básicas y fáciles de conseguir por medios naturales e incluso artificiales (alcohol, drogas, etc.). Por eso, se puede decir que, en rigor, el emotivismo es una especie de vitalismo que desconoce la afectividad del espiritu (Sellés, 2009, p. 182).

En esta misma línea, Polaino-Lorente escribe que "La opción por el instante, o mejor por el placer de cada instante, frustra y reprime en el hombre su capacidad de compromiso. La cultura del instante no es compatible con la cultura del compromiso" (s.f., p. 174).

Se puede afirmar, entonces, que muchos de los problemas que minan la sociedad -aborto, divorcio, ruptura de amistades, falta de compromiso laboral, etc.- evidencian que el hombre actual mira más a la inmediatez del ahora, que al futuro (Sellés, 2009, p. 185). Este vivir relegando proyectos de largo alcance que implican esfuerzo y tenacidad, es signo de preocupación en orden al crecimiento humano personal.

Así, es de resaltar el trabajo llevado a cabo por María Calatrava (2010) sobre los factores determinantes de los estilos de vida y de las opiniones sobre temas de amor, afectividad y sexualidad de los jóvenes españoles. Al indagar sobre las características personales del joven español —entre los 16 y 20 años-, la investigación encontró, en relación con la formación de la voluntad, que "el 14,3\% de los jóvenes refiere que no suele terminar las cosas o proyectos que empieza. El 60,8\% señala que tiende a querer conseguir las cosas inmediatamente y un $18,7 \%$ de los jóvenes afirma que no suele pensar y planificar su futuro" (p. 63). Con la información que apor- tan dichas variables, la investigación afirma "que un $72 \%$ de los jóvenes encuestados tendrían, al menos, uno de los tres rasgos de carácter impulsivo" (p. 63).

Los datos apenas citados, nos dejan ver la influencia de una cultura inmediatista en la configuración del carácter del joven, por tanto, la carencia de una formación para el esfuerzo, para el trabajo constante en la obtención de resultados positivos a largo plazo. Estos hallazgos son reforzados por otras investigaciones con población joven adolescente - 13 a 18 años - tanto en el Perú (Corcuera, de Irala, Osorio y Rivera, 2010) como en El Salvador (de Irala, López del Burgo, Carlos y Calatrava, 2008).

Con respecto a las razones que manifiestan los jóvenes españoles para haber tenido su primera relación sexual, por ejemplo, se deja ver la falta de autonomía personal en la toma de este tipo de decisiones; lo anterior se evidencia en la presión que puede ejercer el grupo de amigos, así como en la influencia de la "pseudocultura del placer y del instante", a la que aludíamos anteriormente, y que viene permeando la sociedad entera. Así, la investigación revela que el 78,1\% de los jóvenes inicia relaciones sexuales por razones asociadas, o bien a la presión de su entorno, como el "no querer ser diferente", "no saber decir que no", "pensar que su pareja le dejaría", "querer ser más popular"; o bien, por falta de control sobre sí mismo y la situación, ya sea "como consecuencia de haber bebido o tomado drogas", "de ver imágenes de sexo" o "por dejarse llevar de la excitación física del momento sin que fuera su intención inicial" (Calatrava, 2010, p. 74).

Otros datos relevantes con respecto al "diagnóstico" que venimos realizando, los encontramos en la asociación entre "actividad sexual precoz" y "derroche del tiempo libre" o realización de "actividades de ocio no constructivas". La investigación actual consultada (De Irala et al., 2008; Corcuera et al., 2010; Calatrava, 2010) arroja una relación significativa entre "haber tenido la primera relación sexual" y una mayor frecuencia de salidas a disco- 
tecas, bares, centros comerciales, jugar videojuegos y conectarse a Internet. Es de notar también que dichos jóvenes puntúan más alto en el consumo de sustancias tóxicas como tabaco, alcohol y otras drogas, al tiempo que no poseen hábitos de ocio saludable como practicar algún deporte, participar en actividades de formación extracurricular — charlas, cursos, catequesis-, visitar museos, ir al teatro, etc.; todas ellas palestra de una formación disciplinada, exigente y sanamente gratificante.

Si dirigimos la mirada concretamente a Latinoamérica, encontramos que el $52 \%$ de los jóvenes adolescentes peruanos, por ejemplo, nunca han formado parte de un voluntariado o realizado diversas actividades de formación artística o cultural (Corcuera et al., 2010). El caso de El Salvador es muy semejante, la investigación arroja que la actividad que más realizan los jóvenes durante su tiempo libre es ver la televisión, escuchar música o leer revistas; además, la mayoría de ellos manifiesta que tienen mucho tiempo libre pero no saben qué hacer con él (De Irala et al., 2008, p. 73). Es fácil de concluir que este tipo de ocio anula la voluntad del joven, puesto que dichas actividades no requieren de él un mayor esfuerzo. Los resultados para España son parecidos, "más de dos tercios de los jóvenes españoles refieren que nunca han hecho voluntariado social y más de la mitad no han realizado actividades de tipo artístico o participado en cursos culturales o formativos" (Calatrava, 2010, p. 127).

Por otra parte, en un estudio de la American Psychological Association (2012), se relacionan numerosas investigaciones sobre la fuerza de voluntad y el autocontrol, llevadas a cabo en niños, adolescentes y adultos. Entre los hallazgos reportados se señala como un dato común la importancia de la fuerza de voluntad o autodisciplina para alcanzar y mantener un buen rendimiento académico en adolescentes, así como el desarrollo de hábitos saludables - se tiene menos problemas de consumo de alcohol y sustancias psicoactivas, por ejemplo-, una mayor autoestima y el establecimiento de relaciones personales más enriquecedoras.
Los hallazgos citados son un indicador de la urgencia de la educación para el esfuerzo y la generosidad, puesto que en el proceso hacia la madurez humana personal, los jóvenes necesitan experimentar la alegría de trabajar por el bien del prójimo, a la vez que desarrollar, en ese darse a los demás, sus capacidades físicas, intelectuales, artísticas y espirituales. Todo lo anterior no se logra sin una pedagogía del esfuerzo o del trabajo bien hecho, del compromiso con el propio crecimiento personal en todos los ámbitos de la vida - afectivo, familiar, de estudio o de trabajo profesional-.

La exigencia y la reflexión parecen ser dos requisitos indispensables para educar la voluntad de niños y jóvenes (Morales, 2008; de Gregorio, 2007). No es extraño, entonces, que una sociedad que fomenta lo espontáneo e impulsivo como signo de autonomía e independencia, adolezca de jóvenes con conductas desadaptativas, donde lo que prima es el acatamiento de sus deseos o tendencias, en lugar de una voluntad inteligente - permeada por la reflexión-con fines claros y constructivos.

El precio que se paga por la difusión del permisivismo y del emotivismo en la familia y la sociedad es alto: la plenitud y felicidad humanas pueden verse comprometidas. "El hombre sin vínculos ni compromisos, ciertamente podrá embriagarse con muchas experiencias placenteras instantáneas, pero solo en la medida en que renuncie a ser feliz" (PolainoLorente, s. f., p. 174). Los educadores deberán repensar quizás las palabras de un conocido educador español, Abilio de Gregorio, al abordar el tema de la exigencia en la formación de jóvenes: "No hay manera de formar el carácter y la voluntad sin una comprensión honda del dolor y del sacrificio" (2007, p. 119).

\section{Fundamentación teórica}

La voluntad es la facultad de obrar persiguiendo un fin reflexivo. En esto se distingue de la terquedad o del capricho, los cuales responden a motivos a menudo instintivos (Peñacoba, 2014). Así, lo que la 
voluntad humana tiene de excelsa lo tiene también de difícil: se consigue con la ayuda de la educación.

Ahora bien, al referirnos a la educación, hemos de tener en cuenta con Maritain (1943) que "El objeto de la educación es guiar al hombre en su desarrollo dinámico, en cuyo curso se forma como persona humana, provista de las armas del conocimiento, de la fuerza del juicio y de las virtudes morales" (p.10). O con García Ramos (1991), cuando afirma que la tarea de la educación es potenciar la persona que es cada uno.

Por tanto, la labor educativa debe obrar sobre el intelecto, la voluntad y también el corazón, pues lo científico no se edifica solo por la inteligencia, sino también por una voluntad orientada por una conciencia bien formada (García Ramos, 1991).

Acerca de lo que es la voluntad y en relación con la capacidad de educarla, señalan Yepes y Aranguren (1996) que es una función intelectual cuyo acto propio es querer y que está abierta al bien. Actúa simultáneamente con la razón.

Ahora bien, podríamos preguntarnos cómo educarla. En la conferencia "Los fines de la educación", Maritain (1943) señala que en primer lugar se debe iluminar y fortificar la razón, para ejercer de ese modo una influencia indirecta sobre la voluntad. Pero añade:

La inteligencia es en sí misma más noble que la voluntad, porque su actividad es más inmaterialy universal. Pero [...] en lo concerniente a las cosas u objetos mismos sobre los cuales recaen nuestras actividades, es mejor querer y amar el bien que simplemente conocerlo. Además, es por su voluntad, cuando es buena, y no por su inteligencia, por perfecta que sea, por la que el hombre se torna bueno (p. 21).

Aparece otro elemento que influye en este proceso educativo: se trata de la fuerza motivadora. La voluntad se dirige hacia algo en cuanto lo reconoce como bueno. El querer en que consiste la voluntad es siempre, además de un querer intelectualizado, un querer motivado.

Con todo, Marina (2009) invita a no quedarse solo en un "motivo" externo para actuar. En la educación es importante la motivación, pero no es suficiente con ella para explicar el comportamiento humano: "En efecto, sería estupendo que todos actuáramos con entusiasmo y motivación, pero no siempre funcionamos así. Hay muchas acciones que debemos realizar, aunque no estemos motivados para ello" (p. 15).

Vale aclarar entonces qué es lo que puede motivar auténticamente una acción. Polaino-Lorente, en su trabajo sobre las dimensiones de la voluntad (1988), diferencia "motivaciones" de "móviles" —entendidos estos últimos como intereses, incentivos o gratificaciones-. Las primeras son los valores reconocidos como tales por el intelecto. Los móviles también mueven a la acción, pero la apelación se dirige hacia aquellas áreas más periféricas y sensibles de la persona: sus tendencias o ciertos tipos de intereses. Mientras las motivaciones se corresponden con la voluntad, los móviles se refieren a los afectos o deseos.

A nivel del comportamiento, se puede decir que, en el acto voluntario, es el sujeto el que se mueve hacia el fin, eligiéndolo; en los deseos de la afectividad, el hombre es "movido" por el objeto de sus deseos casi inconscientemente.

Este peligro de dejarse llevar el hombre por los deseos de la afectividad, es señalado Gordillo al afirmar que:

El querer de la voluntad, es querer algo que ya conoce, lo mismo pasa con el deseo pues para desear algo, tenemos antes que conocerlo, aunque sea de forma confusa o imaginaria. El problema es que nos dejemos llevar por el deseo, con falta de conocimiento, porque los deseos nos conducen a otros deseos, la dinámica del deseo es imparable (2015, p. 61). 
Ante estas tensiones siempre presentes es muy importante tener en cuenta que la elección humana, antes que recaer sobre una cosa u otra, recae sobre la propia persona. Por tanto, por el querer, el hombre construye su biografía; sus actos volitivos van narrando lo que el hombre va haciendo de él mismo en cada acto libre. De ahí que Morales pueda afirmar de manera rotunda que: "La mayor desgracia de un joven, más que la falta de salud o de memoria, es una voluntad débil. Su mayor fortuna, más que un gran talento, es una voluntad firme y tenaz" (1999, p. 174). $\mathrm{El}$ acto voluntario es, entonces, un acto previsto, premeditado y, por ello, libre (Polaino-Lorente, 1988).

En adolescentes o jóvenes estudiantes que no han formado su voluntad es común encontrar que su conducta deviene en "respuestas automáticas" suscitadas por los impulsos del momento. De ahí que distintos autores enfaticen la necesidad de permear las decisiones con la reflexión y el silencio para hacerse plenamente responsable de lo elegido. Así se lee en el estudio titulado Ideología y ética de la voluntad: "La toma de conciencia de mis acciones voluntarias necesita una fundamentación del querer [...] No podemos comprometernos con nuestras elecciones si no somos conscientes de nuestro juicio de conciencia" (Gordillo, 2015, p. 59).

Como señala Peñacoba (2014) en su investigación sobre educación de la libertad, una de las posibilidades de la voluntad es tanto el inhibir pulsiones poco deseables, como el impulsar iniciativas y proyectos. Por ello, no es conveniente eliminar toda incomodidad en el orden material y toda inhibición en el comportamiento, pues ante las dificultades es necesario, por un lado, resistir, y por otro, tener iniciativa para afrontar la dificultad. Es así como se adquiere "fuerza de voluntad".

Lo afirma García Hoz: "Lo específico de la formación de la voluntad se apoya en la habituación práctica, es decir en el desarrollo de la capacidad de esfuerzo - aplicación de la energía moral- que se halla a la base de la actividad humana" (1988, p. 89).
Es entonces la voluntad la que permite el autodominio. Gracias a la voluntad, en el hombre se da la autoposesión de sí mismo y la capacidad de autoconducirse con independencia de los estímulos ambientales o de los objetos hacia los que se experimenta atracción o rechazo. La educación de la voluntad es educación para la libertad en los jóvenes, en cuanto agudiza el sentido crítico de la inteligencia que juzga cada vez más óptimamente y en cuanto facilita cada vez más la plena posesión personal en el obrar diario.

Los educadores tienen un papel irrenunciable en este camino de crecimiento humano personal por medio del desarrollo de la voluntad libre. Así escribe Barrio Maestre:

Cuando se es muy pequeño se carece aún de esa capacidad de autodeterminación hacia algo que cuesta un cierto esfuerzo, y entonces se necesita quizá que otras personas mayores -los educadores, padres y maestros - empujen un poco al comienzo. Los educadores han de ser conscientes de que esa ayuda es necesaria, aunque sea algo costoso, sobre todo a ciertas edades más "dificiles" (2007, p. 16).

Lo mismo sugiere Cardona (2001) al indicar que en su tarea el educador debe mostrar al educando el sentido y la finalidad de cada acto, de modo que así se sienta libre ante las distintas normas de conducta que se le exijan.

¿Y qué hacer si se presentan el cansancio y el desánimo en el educando? Jiménez Abad señala que

...el cansancio de la voluntad no se presenta porque surjan los obstáculos, sino porque estos ocultan la meta a nuestra mirada y porque nuestras mejores disposiciones tienen también sus limites. Es preciso entonces recordar y reavivar el sentido de lo que se está haciendo, manteniendo viva la conciencia de lo que se persiguey el valor de lo que se hace (1997, p. 108). 


\section{Tendencias relevantes en educación de la voluntad}

En una primera aproximación a las tendencias educativas sobre la formación de la voluntad de los niños, adolescentes y jóvenes en la sociedad actual, en los ámbitos de la educación formal —instituciones educativas como la escuela y la universidad-y no formal —familia, asociaciones juveniles, etc.se pueden señalar las siguientes: educación de la voluntad propiamente dicha, entendida esta como educación en la virtud -especialmente en los países hispanos-, educación del carácter - desarrollada con singular fuerza en Estados Unidos- y educación moral.

\section{Educación de la voluntad}

En una primera exploración de la educación de la voluntad, se encontró que esta es entendida principalmente como una educación en virtudes y que está inserta, a su vez, en la categoría más amplia de formación integral de la persona.

Al respecto, sobresale la propuesta educativa de Tomás Morales, educador español cuya vida atravesó casi todo el siglo XX (1908-1994) y cuyos principios pedagógicos se encuentran recogidos en un solo volumen, en Obras Pedagógicas (2008). Dada la relevancia de su quehacer educativo en orden a la formación de la voluntad, se le dedica una atención amplia en este artículo.

La novedad de Tomás Morales radica en "ser un pedagogo sin escuela, pero que creó escuela" como afirma Abilio de Gregorio (2007). No fue un pedagogo en sentido formal, pero sí ha propuesto un sistema pedagógico real para el hombre de hoy. Trabajó durante décadas en la formación integral de los jóvenes (1940-1994), y, a través de ellos, su influjo educativo alcanzó la familia, la empresa, la cultura y la enseñanza - escuelas y universidades-. Como afirma Lafuente (2008), se trata de una pedagogía original e innovadora, que tiene por objetivo capacitar a la persona para que se desarrolle integramente, formando a su vez a otros.
La "forja de la voluntad" es su énfasis característico, como él mismo afirmaría en un documento dirigido a los miembros de la obra por él fundada. Así escribe: "No olvidéis nunca que la formación de la voluntad es la característica distintiva en la pedagogía de la Cruzada-Milicia. Sobran ideas, faltan voluntades que las vivan" (Morales, 2006, p. 64).

Van en aumento los estudios realizados sobre su propuesta educativa tanto en Europa (Gazapo, 1997; Gómez, 1998; Zúñiga, 2004; Redondo, 2004; Lafuente, 2007; De Gregorio, 2007; etc.) como en Latinoamérica (Velasco, 2005; Jara, 2009; Cárdenas, 2014).

Así, en la obra monográfica de Abilio de Gregorio (2007), Tras las huellas de la pedagogía del Padre Tomás Morales, se destaca que es la voluntad, dentro de la vida psíquica, la que permite tomar conciencia de ese centro unitario que denominamos "yo". Es por eso precisamente - afirma el autor - que "a mayor voluntad, mayor conciencia de ser persona” (p. 124), de ahí que en el lenguaje común se equipare voluntad con personalidad. "Decimos de alguien que tiene voluntad cuando se manifiesta en su vida la presencia de una instancia superior a sus impulsos páticos que le hace dueño de sí mismo" (p. 124).

Los principios educativos que propone Tomás Morales están orientados a la formación de una minoría selecta de hombres y mujeres que deben ser líderes de la sociedad, pero antes de sus propias vidas. Para ello, Morales los "lanzaba" a "nadar contracorriente", en medio de una sociedad saturada de materialismo, permisiva y facilista. Los estudiosos de su obra aseguran que, así las cosas, su pedagogía debía girar necesariamente en torno a un gozne fundamental: la educación de la voluntad (Del Hoyo, 2009; de Gregorio, 2007; García, 2006; Gómez, 1998, etc.).

Una de las patologías de la voluntad es la indecisión. De ahí que muchas personas no sean capaces de ponerse metas y cumplirlas. Esto ocurre, la mayoría de las veces, por el miedo a la renuncia que significa "elegir algo" en sacrificio de otras posibilidades. Una voluntad así es incapaz de comprometerse (de 
Gregorio, 2007, p. 125). Morales lo expresaba de esta forma: Hoy "tenemos que infundir en el joven el valor de ser uno mismo, de existir personalmente, de no ser una simple emanación del entorno [...] Esta es la condición indispensable para llegar a ser capaz de un compromiso responsable" (2008, p. 127).

Para enseñar a los jóvenes a asumir responsabilidades y tomar decisiones, Morales (2008) propone la pedagogía del "hacer-hacer". Esto implica delegar responsabilidades en el educando, de modo que aprenda a asumir compromisos, con los riesgos que toda decisión libre lleva consigo.

Otro de los factores que influyen en la debilidad de la voluntad es la falta de un horizonte ilusionante que dé sentido a las acciones. El presentimiento de que todo esfuerzo es inútil porque la realidad es hostil, empantana las raíces mismas de la voluntad. Para Morales, el sentimiento vital también se educa: "Domínate y siempre estarás alegre. No olvides que es dentro de nosotros, no fuera donde hace buen o mal tiempo" (2013, p. 72).

Por eso "el educador no debe permitir la flojera del pesimismo que esteriliza la voluntad del joven" (de Gregorio 2007, p. 128), debe proponerle ideales grandes y entusiasmantes. Solo así podrá exigir todo del educando, sabiendo que un joven entusiasmado por lo valioso, más fácilmente da mucho que poco.

Con todo, la fuerza de voluntad no se adquiere instantáneamente al fijarse una meta, precisa la adquisición de hábitos buenos. Para ello Morales propone el "cultivo de los pequeños detalles". "La sujeción a los pequeños detalles va acostumbrando la voluntad del novel deportista al cumplimiento del deber en las grandes y decisivas circunstancias de la vida" (2008, p. 45). Aconseja aficionar al joven a rendir culto al detalle: puntualidad, orden, control de sentidos, espacios de silencio, tener un horario, etc. (Lafuente, 2007).

Aunque la experiencia demuestra que esta conquista de la voluntad es difícil al principio, una voluntad fuerte "es la consecuencia de pequeñas victorias cotidianas en el dominio de uno mismo, y eso es lo que los jóvenes necesitan especialmente hoy en día" (García, 2006, p. 12).

De ahí que uno de sus grandes principios educativos sea, junto al cultivo de la reflexión, la mística de exigencia y el espíritu combativo, la escuela de constancia.

No es suficiente desarrollar en el joven su poder de reflexión. Si no troquela - el educador - su voluntad endureciéndola con el sacrificio, aquellas ideas quedarán para siempre en la fría región de las disquisiciones especulativas sin que desciendan a fecundar la vida [...] La experiencia de largos años me ha revelado que, efectivamente, ante el poder arrollador de una voluntad constante, nada se resiste (Morales 2008, p. 159).

Lo que más contribuye, según Morales, a forjar en el joven una voluntad constante es el yunque del trabajo-estudio, con afán de superar la medianía ambiente que nos envuelve. Así escribe "Esos profesionales (espléndidos) no se improvisan. Son el fruto maduro de años de estudio y de trabajo, de reflexión y de constancia" (2008, p. 191). En este aspecto, Morales engarza con otro gran educador español, García Hoz (1987) y su propuesta-fundamento de la educación de la voluntad, "la obra bien hecha", de la que nos ocuparemos más adelante.

Bienvenido Gazapo (1997) apunta que la acción y la corrección de la acción son los dos ejes insustituibles de la dinámica educativa de Tomás Morales.

Con respecto a la acción, el P. Morales da el siguiente consejo: "No hay que permitir al joven que se entretenga en largas discusiones, sino obligarle a actuar, venciendo dificultades, saboreando alegrías y cosechando experiencias que hagan cada vez más fecundas su acción" (2008, p. 235). Enseñarle también a saber aceptar los aparentes fracasos y a no dejarse bloquear por sus limitaciones. "El único fracaso es no actuar" repetirá constantemente a sus 
educandos, pues tras la acción podrá corregir los posibles defectos cometidos.

El autocorrectivo es esencial en su propuesta educativa. "Una mala costumbre bloquea la voluntad. Lo que intenta el autocorrectivo es liberarla de esa cadena para que pueda seguir actuando" (Morales, 2008, p. 140). Para este gran educador de juventudes, no bastan los buenos propósitos de no volver a caer. De esta manera, no se educa la voluntad, solo el entendimiento. "Los buenos propósitos incumplidos hacen que la conciencia se haga más laxa”, escribirá (2008, p. 140). Pero el autocorrectivo, para que sea eficaz, no puede ser impuesto sino totalmente personal, y debe ir a la raíz del fallo que se quiere erradicar.

A la dinámica de correctivo-autocorrectivo, Morales añade también la del premio y autopremio. Para lograr la armonía en la formación del joven es necesario también reparar las fuerzas gastadas con aquello que al joven le descansa y estimula. Otra ventaja del autopremio es que encamina al joven a un mayor conocimiento de sí y a un aumento de confianza en sus posibilidades (Lafuente, 2007).

Entre los medios con que el P. Morales formó la voluntad de cientos de jóvenes y que los educadores por él formados siguen practicando hoy en día, resaltamos especialmente las residencias-hogares para universitarios y empleados (en un ambiente de austeridad y alegría se promueven la responsabilidad y el autogobierno de la misma residenciahogar), y los campamentos (en el contacto con la naturaleza se cultivan el esfuerzo y la superación, asi como la generosidad y la amistad en la convivencia con otros jóvenes) (Zúñiga, 2004).

Como se puede apreciar, la pedagogía moraliana para educar la voluntad de los jóvenes es una "pedagogía de la virtud" (Zúñiga, 2004).

Otro notable educador español es Víctor García Hoz (1911-1998), quien propone, desde la reflexión teórica, la "pedagogía de la obra bien hecha" como el fundamento de la educación de la voluntad en la vida universitaria (1988).

La Obra Bien Hecha, explícita expresión de Eugenio D'Ors, exige el bien saber y el bien hacer, excelencia intelectual y perfección moral, lo cual constituye la razón de ser de la Universidad. Una obra bien hecha es una obra que abarca el bien en todas sus significaciones: el técnico y el personal.

En un ambiente donde impera la mediocridad, escribe García Hoz,

La Obra Bien Hecha es manifestación de la responsabilidad personal en contraste con la ruindad de la chapuza. Mientras la chapuza responde a la astuta ocurrencia o a la rutina de un trabajo desangelado, la Obra Bien Hecha exige inteligencia para concebirla, aptitud para realizarla y voluntad de acabamiento (1987, p. 66).

García Hoz coincide con Morales no solo en los aspectos citados, sino también en la capacidad formadora de la valoración, última etapa formativa de la obra bien hecha. La valoración, por parte del educador y de la comunidad, así como la autovaloración, llevada a cabo por el propio educando, buscan rectificar o mejorar el trabajo realizado. La alegría fruto de la obra bien hecha es el mejor aliciente en este camino de formación emprendido.

En la misma línea de las propuestas formativas adelantadas por Morales y García Hoz, encontramos las reflexiones desarrolladas por José María Barrio (2007) "La persona humana es más cuanto más y mejor conoce y quiere. Ser persona significa siempre poder ser más" (p. 14).

Este crecimiento se da a través de la adquisición de hábitos intelectuales y morales que lo capacitan para gestionar adecuadamente su libertad. Así escribe el filósofo español: "La maduración de una persona estribará, entonces, en la provisión de hábitos que la perfeccionan en su entender y en su querer, que le ayudan a pensar y actuar ordinariamente bien" (Barrio, 2007, p. 15). 
El desarrollo de hábitos virtuosos implica fuerza de voluntad, pero son precisamente estas virtudes las que hacen, por un lado, "habitable" nuestra existencia y nos dotan de libertad.

Al respecto, escribe Barrio Maestre:

Las costumbres firmemente asentadas en nuestra vida le suministran un cierto arraigo y cobijo, una bóveda axiológica que nos protege de la intemperie y permite que nos sintamos en nuestro sitio [...] Por el contrario, para la persona que carece de pautas estables de pensar y actuar — de "criterio"- su vida está hecha de improvisaciones y bandazos: resulta inhabitable por incoherente, inaferrable e indisponible (2007, p. 18).

Para cerrar este apartado, y de manera muy breve, mencionaremos los aportes teórico-prácticos de Ouintana Cabanas, Otero Cama y Rojas.

Quintana Cabanas (1995), en su obra Pedagogía moral, afirma que una serie de elementos contribuyen a conseguir una mayor fuerza de voluntad (esfuerzo, frecuencia en hacer actos de voluntad, ideales, motivaciones fuertes). Indica también factores que pueden estorbar la voluntad (desinterés, actuar de un modo irreflexivo, insensibilidad, exceso de deliberaciones minuciosas ante la decisión, demasiada influencia de un líder o de un grupo determinado, inmadurez personal que somete a la persona a sus impulsos del momento).

Otero Cama (1998), por su parte, señala también algunas propuestas educativas para el perfeccionamiento de la voluntad. Su investigación parte de que "perfeccionar la facultad volitiva del hombre implica mejorar a la persona en su globalidad” (p. 97).

Desarrolla en su propuesta aquellos factores asociados con el perfeccionamiento de la voluntad y que deben ser tenidos en cuenta por las instituciones educativas que pretendan trabajar en esta línea. Señala una adecuada jerarquización de valores, la valoración de las situaciones de esfuerzo en el edu- cando, y crear situaciones concretas para el fomento y el desarrollo de virtudes humanas.

Del mismo modo, Enrique Rojas (2010), gran estudioso e impulsor de la educación de la voluntad, se refiere a diez reglas para tener en cuenta en la educación de la misma. Contar con ellas es garantía de una eficaz educación de la voluntad.

\section{Educación del carácter}

Otra de las tendencias referidas es la educación del carácter. Así, "educación del carácter se usa en Estados Unidos como un término general que agrupa iniciativas diversas, vinculadas con la educación en virtudes, educación en valores, educación para la ciudadanía, educación para la construcción personal y educación de la afectividad" (Vargas y González-Torres, 2009, p. 1381).

En las propuestas más genuinas, la educación del carácter se dirige a conocer, amar y obrar el bien.

Robert Roberts, uno de los más prestigiosos expertos en este tema por liderar el Jubilee Centre for Character and Virtues (JCCV), a la pregunta ¿cómo definir la educación del carácter?, responde que esta "es en gran medida una educación sentimental, es decir, una formación de la voluntad y de las emociones, y también del entendimiento" (Bernal, Gonzáles-Torres y Naval, 2015, p. 41). Por su parte, Randall Curren, en la misma publicación científica, considera que, aunque no tiene una definición como tal de la educación del carácter, esta "incluye la formación del entendimiento, las virtudes y las capacidades esenciales para el buen vivir" (p. 41).

En un estudio sobre la educación del carácter (Vargas y González-Torres, 2009) se concluye que, después de un cierto declive en Estados Unidos, hacia finales del siglo XX se ha puesto en marcha el movimiento de la revitalización del carácter (MRC) que relanza esta educación en su concepción aristotélica como educación en las virtudes. El MRC busca reinsertar en el ámbito escolar y en la investigación 
la noción clásica de la educación del carácter por la virtud y responder asía los problemas de la juventud que no habían conseguido satisfactoria respuesta con los programas educativos anteriores - como la Teoría del desarrollo moral cognitivo de Kohlberg, entre otros-. Una vez más, para Robert Roberts, el resurgir de la educación del carácter en la actualidad es una "respuesta a una insatisfacción con la educación convencional, y a la sensación de que una educación limitada a la información y a habilidades técnicas no es una educación real ni completa" (Bernal, Gonzáles-Torres y Naval, 2015, p. 41).

La educación del carácter en los últimos años se ha visto fortalecida por la naciente psicología positiva. "Había que empezar a ver a los jóvenes más 'como recursos' que como 'problemas'y adoptar una perspectiva multicausal y ecológica (implicar a las escuelas, familia y comunidad más amplia en el desarrollo positivo de los jóvenes)" (Vargas y GonzálezTorres, 2009, p. 1392).

Por otra parte, en un interesante análisis que hace Mesa (2004) de la educación del carácter, muestra cómo se remonta a Aristóteles y luego a la escolástica. Señala que se agrupan en este concepto, por un lado, el aprendizaje de las virtudes de las generaciones anteriores, que se inspira en la visión Aristotélica de la vida buena como práctica habitual de las virtudes. Sobre qué virtudes se han de cultivar varían los distintos autores: respeto y responsabilidad (Lickona, 2004a); humildad, prudencia y lealtad para los adolescentes (García, 2006). Mesa (2004) señala también que la educación del carácter representa la visión opuesta a la clarificación de valores — dentro del desarrollo moral cognitivo de Kohlberg-, pues esta postura empuja hacia el individualismo.

En este contexto, es de resaltar el documento de 1996, Character Education Manifesto, cuya finalidad era unificar los principios centrales de la educación del carácter (EC). Fue el primer documento que planteó una definición común de la noción de carácter y propuso además ocho principios guía para las reformas educativas para implantar en los programas educativos.

Como se puede apreciar, en este campo aparece un abanico de experiencias educativas de diferente talante. Se destacan en este campo los aportes de Thomas Lickona. Detallaremos por su importancia su propuesta. Lickona comprende la educación del carácter como el cultivo de la virtud. En uno de sus libros (2004a), señala que es necesario que el centro educativo se convierta en un lugar donde el carácter sea lo primordial. Para conseguirlo influye el ejemplo, la relación entre maestros, la disciplina, el currículo, los estándares académicos, la ética del ambiente del centro, las actividades extracurriculares y el involucramiento del personal —académico, administrativo, alumnos y padres-.

De un modo más concreto, en Convierte tu escuela en una escuela de carácter, Lickona (2004b) señala todo un proceso para la educación del carácter, incluyendo un ideario, máximas, escoger una estrategia organizada que promueva las virtudes, realizar el seguimiento y evaluación del plan, construir una comunidad fuerte de adultos, etc. Por otra parte, Lickona (2004b) muestra más de un centenar de estrategias que padres y escuelas pueden usar para ayudar a los jóvenes a construir un carácter personal adecuado.

Aunque ciertamente hay una gran diversidad en la manera de entender la educación del carácter, llevando con ello a no poca confusión a la hora de realizar un programa concreto de educación en este orden, Lickona de manera rigurosa precisa qué es el carácter y cómo hay que educarlo. Así:

El carácter es el conjunto de hábitos de la mente, del corazón y de la conducta; entendiendo por hábito una disposición interna, y relativamente estable, que capacita para responder a las situaciones de una forma moralmente buena. En esencia, apunta que un buen carácter consiste en conocer el bien, amar el bien y hacer el bien. 
De este concepto de carácter deriva la consideración de la EC como el esfuerzo deliberado por enseñar virtudes (Bernal, Gonzáles-Torres y Naval, 2105, p. 43).

Hernández-Sampelayo (2007) hace un interesante análisis de la aplicación de la formación del carácter en la misma línea expuesta. Así, menciona los programas de educación ciudadana en los colegios Blue Ribbon (Cinta Azul). O también el programa Core Virtues, en el cual se pretende que los alumnos se enamoren del bien y la virtud a través de buena literatura y cultivando cada mes una virtud específica.

Como vemos, hay una vitalidad muy grande en las aplicaciones de la educación del carácter. Todas estas propuestas inciden de un modo u otro en el fortalecimiento de la voluntad de los educandos, de ahí que se configuran como una tendencia educativa actual en este orden.

\section{Educación moral}

En la tercera variante se estima la educación de la voluntad inserta en la educación moral.

Así, Berkowitz (1995) indica que "necesitamos crear programas educativos morales para promover el desarrollo de personas morales completas, con carácter, razonamiento, valores, emociones y conducta" (p. 96).

Por su parte, Mesa (2004) realiza un recorrido crítico por cuatro de las propuestas morales más influyentes: la clarificación de valores, la educación del carácter, el desarrollo moral cognitivo de Kohlberg -que presenta dos propuestas diferentes: discusión moral y escuela justa- y la propuesta feminista de la educación del cuidado.

Afirma que los enfoques de educación moral presentan distintas soluciones a la problemática del individuo y la comunidad. Así, en la clarificación de valores tiene un predominio considerable la au- tonomía personal. Por su parte, en la educación del carácter se encarna la visión que podemos llamar tradicional. Kohlberg, a su vez, encarna un individualismo liberal en la discusión moral, mientras que con la escuela justa se reencuentra con la dimensión comunitaria. Finalmente, la propuesta feminista de Gilligan y Noddings busca resaltar el valor de lo relacional.

Es interesante también lo que propone García Ramos (1991) cuando aconseja a los universitarios el razonamiento, el cultivo de la creatividad y los valores estéticos, de la cultura y la solidaridad. Les anima también a la autenticidad y a potenciar el ser por encima del tener o del hacer, superándose cada día.

Así, tal como se ha expuesto, hay intentos englobados en la educación moral que se ocupan de la formación integral de los jóvenes, de modo que contemplan también de algún modo la educación de la voluntad.

\section{Conclusiones}

La educación de la voluntad en el joven es imprescindible hoy, pues favorece en él un crecimiento personal. Por ello, las instituciones educativas deben velar por facilitarle esa ayuda que le permita decidirse por lo bueno y valioso, frente al permisivismo relativista y emotivismo actuantes en la sociedad de hoy.

No es fácil la tarea. Será preciso cultivar, junto con las virtudes en general, la reflexión y el esfuerzo constantes como requisitos indispensables en la formación de la voluntad. Ellos permitirán saber orientar las propias tendencias y pasiones por medio de una voluntad inteligente que tiene fines claros y constructivos.

Así, para el joven será mucho más sencillo, entonces, valorar cualquier opción, sopesando sus ventajas e inconvenientes, lo que es propio además del hombre prudente. Podrá así asumir la responsabilidad de sus actos y la orientación de su vida. 
La persona así educada sabrá distinguir entre las motivaciones que su voluntad le presenta y los móviles o intereses que, casi inconscientemente, generan las emociones o las propias tendencias, suplantando en ocasiones la actuación libre.

De este modo, el ser humano construye su propia biografía a través de los actos voluntarios. Así, por la voluntad se da en él la autoposesión de sí mismo junto con la posibilidad de conducirse libre y responsablemente en la vida.

En este proceso tienen un papel irrenunciable los educadores - tanto en la educación formal como en la no formal e informal-. Así lo muestran muchos de los ejemplos que se señalan en la última parte de este artículo, al amparo de las tendencias reconocidas y expuestas en el mismo.

Así, tanto con iniciativas que enfocan la formación de la voluntad como educación de la virtud, como con aquellas que abarcan la educación del carácter (en cuanto educación en las virtudes, en los valores, para la ciudadanía y en la afectividad), o con las que se centran en una educación moral como formación integral, se muestra la necesidad de incidir en la educación de la voluntad, así como la conveniencia de desarrollar nuevas investigaciones que propendan al diseño de estrategias pedagógicas para la formación de la voluntad de los jóvenes en la sociedad actual.

\section{Referencias}

American Psy chological Association (2012). Lo que debemos saber sobre la fuerza de voluntad: la ciencia psicológica del autocontrol. Recuperado de https://www.apa.org/helpcenter/willpower-spanish.pdf

Barrio, J. M. (2007). Cómo formar la segunda naturaleza. Notas antropológicas acerca de la educación de los hábitos. ESE. Estudios sobre Educación, 13, 7-23.

Berkowitz, M. W. (1995). Educar la persona moral en su totalidad. Revista Iberoamericana de Educación. Educación y Democracia, 8, 73-101. Recuperado de http://www.rieoei.org/oeivirt/rieo8ao3.htm

Bernal, A., González-Torres, M.C. y Naval, C. (2015). La educación del carácter. Perspectivas Internacionales. Participación educativa. Revista del Consejo Escolar del Estado, 4 (6), 35-45.

Calatrava, M. (2010). Determinantes de los estilos de vida y las opiniones de los jóvenes españoles sobre afectividad, amor y sexualidad [Tesis para obtener el título de doctor] Universidad de Navarra: Facultad de Medicina.

Cárdenas, A. M. (2014). Educar la afectividad mediante la experiencia estética. Percepciones e indicaciones a la luz de los planteamientos pedagógicos de Tomás Morales y Alfonso López Quintás. Revista arbitrada del Centro de Investigación y Estudios Gerenciales A.C., 5 (1), 341-371. Recuperado de http://www.grupocieg.org/ archivos_revista/5-1-22\%2O(341-371)\%20C\%C3\%A1rdenas\%2ORuiz\%2OAgosto\%2014_articulo_id149.pdf

Cardona, C. (2001). Ética del quehacer educativo. Madrid: Rialp.

Center for the Advancement of Ethics and Character (1996). Character Education Manifesto. Boston University School of Education. Recuperado de http://eric.ed.gov/?id=ED407299 
Corcuera, P., de Irala, J., Osorio, A. y Rivera, R. (2010). Estilos de vida de los adolescentes peruanos. Lima: Aleph.

De Irala, J., López del Burgo, C., Carlos, S. y Calatrava, M. (2008). Adolescentes con cultura. Estilo de vida de los estudiantes adolescentes de El Salvador. El Salvador: Concultura.

De Gregorio, A. (2007). Por las huellas de la pedagogía del padre Tomás Morales, un idealista con los pies en la tierra. Madrid: Fundación Universitaria Española.

De Gregorio, A. (2008). La formación de la personalidad en la pedagogía del Padre Tomás Morales: educar para una vida creadora. Cuadernos de Pensamiento, 21, 285-312.

Del Hoyo, J (2009). Profeta de nuestro tiempo (Tomás Morales SJ, 1908-1994) (2 ed.). Madrid: Ediciones Encuentro.

García, G. (2006). Tomás Morales and the Integral Education of Young People: The Milicia de Santa Maria [Tesis para obtener el título de Master en Artes y Educación]. Dublin: The National University of Ireland University College Dublin.

García, J. M. (1991). La formación integral: objetivo de la Universidad. Revista Complutense de Educación, 2 (2), $323-335$.

García, V. (1987). La formación del universitario en la obra bien hecha. Cuadernos de pensamiento, 2, 59-66. Recuperado de http://www.fuesp.com/pdfs_revistas/cp/2/cp-2.pdf

García, V. (1988). La obra bien hecha, fundamento de la educación de la voluntad. En VV. AA. Dimensiones de la voluntad (pp. 89-97). Madrid: Dossat.

Gazapo, B. (1997). Tomás Morales, forjador de hombres. Aproximación a su estilo educativo. Madrid: Ediciones Encuentro.

Gómez, M. E. (1998). Una pedagogía para el hombre de hoy. Madrid: Fundación Universitaria Española.

Gordillo, L. (2015). Ideología y ética de la voluntad. Una base para la reflexión bioética. Cuadernos de Bioética, $26(1), 51-65$.

Hernández-Sampelayo, M. (2007). La educación del carácter. Madrid: Eiunsa.

Jara, A. (2009). Elaboración de una propuesta para la Educación de la Voluntad de las jóvenes estudiantes del $5^{\circ}$ grado de Educación Secundaria de la I.E.N. Rosa Flores de Oliva, siguiendo los Principios Pedagógicos del P. Tomás Morales Pérez en el año 2008 [Tesis de licenciatura en filosofía y religión]. Chiclayo: Universidad Santo Toribio de Mogrovejo.

Jiménez, A. (1994). Un aspecto concreto: la formación de la voluntad. En García, V. La orientación en la educación institucionalizada. La formación ética (pp.100-125). Madrid: Rialp.

Jiménez, A. (1997). Valores y maestros. Cuadernos de Pensamiento, 11, 101-110. Recuperado de: http://www.fuesp. com/pdfs_revistas/cp/11/cp-11.pdf 
ISSN $0123-1294$ | e-ISSN 2027-5358 | Educ.Educ. Vol. 19. No.3 | Septiembre-Diciembre de 2016 | pp. 439-457.

Universidad de La Sabana | Facultad de Educación

Jiménez, A. y Sánz, A. (2015). Hábitos y valores: un área de mejora para los centros. Participación educativa. Revista del Consejo Escolar del Estado, 4 (6), 81-89.

Lafuente, B. (2007). La pedagogía del P. Tomás Morales, como formación integral. Una respuesta realista a los retos educativos actuales [Tesis doctoral]. Barcelona: Universidad de Barcelona.

Lafuente, B. (2008). Formación realista e integral de la persona en la pedagogía del P.Tomás Morales. Edetania: estudios y propuestas socio-educativas, 35, 199-208.

Lickona, T. (2004a). Character Matters: How to Help Our Children Develop Good Judgment, Integrity, and Other Essential Virtues. New York: Simon and Schuster.

Lickona, T. (2004b). Convierte tu escuela en una escuela de carácter. Recuperado de http://www.hazpolitica.org/ noticias/index.phtml?id=461

Marina, J. A. (1997). El misterio de la voluntad perdida. Barcelona: Anagrama.

Marina, J. A. (2009). Educación del carácter. Núcleo de la personalidad. Cuadernos de Pedagogía, 396, 12-15. Recuperado de http://www.educacion.navarra.es/documents/27590/203401/Educacion_caracter+MARINA. pdf/b8a65fo2-a33d-44a5-82bd-a51f1854e3a8

Maritain, J. (1943). Los fines de la educación. Recuperado de http://www.jacquesmaritain.com/pdf/10_ EDU/O1_ED_FinEdu.pdf.

Mesa, J. A. (2004). Tendencias actuales en la educación moral. Revista Latinoamericana de Ciencias Sociales, Niñez y Juventud, 2 (1), 9-26. Recuperado de http://revistaumanizales.cinde.org.co/index.php/Revista-Latinoamericana/article/view/322/190

Morales, T. (1999). Pensamientos. Burgos: Amábar.

Morales, T. (2006). Vademecum. Madrid: Cruzadas de Santa María.

Morales, T. (2008). Vida y Obra de Tomás Morales, SJ. Obras Pedagógicas, 2. Madrid: BAC.

Morales, T. (2013). Coloquio familiar. Madrid: BAC.

Naour, A. (2005). Padres permisivos, hijos tiranos. Barcelona: Ediciones B.

Osorio, A. (2010). Insuficiencia de los constructos psicológicos en la educación del altruismo. Educación y educadores, 13 (1), 125-138

Otero, F. (1998). Los factores asociados al perfeccionamiento de la voluntad. Educación y educadores, 2, 97-102.

Peñacoba, A. (2014). Educación de la libertad y plenitud personal en Millán - Puelles. Hacia el perfeccionamiento. Saarbrûcken, Alemania: Publicia.

Polaino-Lorente, A. (1988). Dimensiones motivacionales y cognoscitivas de la educación de la voluntad. En VV. AA. Dimensiones de la voluntad (pp. 71-88). Madrid: Dossat. 
Polaino-Lorente, A. (s.f.) La cultura del instante. Recuperado de https://buenosarticulos.files.wordpress.com/2011/ 03/la-cultura-del-instante.pdf

Quintana, J. M. (1995). Pedagogía moral. El desarrollo moral integral. Madrid: Dykinson.

Redondo, L. (2004). “El más”. Duc in altum. Idealismo y realismo en pedagogía del P. Morales. En Profeta de nuestro tiempo. P. Tomás Morales. Actas del Primer Congreso Internacional (pp. 162-170). Madrid: Cruzados de Santa María.

Risco, A., Sánchez, A. y Urchaga, J. D. (2010). Pedagogía del sentido de la vida en el ámbito educativo: estudio empírico con jóvenes Salmantinos. Nous. Boletín de logoterapia y análisis existencial, 14, 33-46.

Rojas, E. (2010). La conquista de la voluntad. Cómo conseguir lo que te has propuesto. Madrid: Planeta.

Sacristán, D. (1988). La importancia del esfuerzo en el proceso educativo. En VV. AA. Dimensiones de la voluntad (pp. 115-141). Madrid: Dossat.

Sellés, J. (2009). La enfermedad mortal del emotivismo. En Jiménez, L. (dir.). La juventud a examen (pp. 167-194). Madrid: Fundación Universitaria Española.

Vargas, L. y González-Torres, M. C. (2009). La revitalización de la Educación del Carácter en el ámbito psicoeducativo actual: aportaciones desde las ciencias de la Prevención y la Psicología Positiva. Electronic Journal of Research in Educational Psychology, 7(19), 1379-1418.

Velasco, M. D. (2005). Los principios pedagógicos del Padre Tomás Morales. Una propuesta para su aplicación en la pastoral universitaria [Tesis de Licenciatura en Educación]. Perú: Pontificia Universidad Católica del Perú.

Yepes, R. y Aranguren, J. (1996). Fundamentos de antropología. Un ideal de la excelencia humana (4 ed.). Pamplona: Eunsa.

Zúñiga, K. (2004). La pedagogía de la virtud en el P.Tomás Morales. En Profeta de nuestro tiempo. P. Tomás Morales. Actas del Primer Congreso Internacional (pp. 171-179). Madrid: Cruzados de Santa María. 
